\title{
Intuitionistic anti fuzzy normed ideals
}

\author{
Nour Abed Alhaleem ${ }^{1}$ and Abd Ghafur Ahmad ${ }^{2}$ \\ ${ }^{1}$ Department of Mathematical Sciences, Faculty of Science and Technology \\ University Kebangsaan Malaysia, Bangi 43600, Malaysia \\ e-mail: noorb@ymail.com \\ 2 Department of Mathematical Sciences, Faculty of Science and Technology \\ University Kebangsaan Malaysia, Bangi 43600, Malaysia \\ e-mail: ghafur@ukm. edu.my
}

Received: 1 February 2021

Accepted: 20 March 2021

\begin{abstract}
In this paper, we introduce the notion of intuitionistic anti fuzzy normed subrings and intuitionistic anti fuzzy normed ideals and various properties of intuitionistic anti fuzzy normal subrings and ideals are examined. Also, we prove that the intersection of two intuitionistic anti fuzzy normed ideals is also an intuitionistic anti fuzzy normed ideal. Besides, we define the anti level subsets and identify the relationship between these sets and intuitionistic anti fuzzy normed ideals. In addition, we define the anti-image of intuitionistic anti fuzzy normed ideals and discuss the inverse image and anti-image for intuitionistic anti fuzzy normed ideals under epimorphism mapping.
\end{abstract}

Keywords: Intuitionistic fuzzy normed subrings, Intuitionistic anti fuzzy normed subring, Intuitionistic anti fuzzy normed ideals, Intuitionistic anti fuzzy normed normal ideals, Anti image. 2020 Mathematics Subject Classification: 03F55, 03E72.

\section{Introduction}

After the introduction of fuzzy sets by Zadeh [21] several researchers investigated on the generalization of the concept of fuzzy set. In 1971 [17], Rosenfeld initiated the studies of fuzzy group theory by introducing the concepts of fuzzy subgroupoid and fuzzy subgroup. Later in 1982, Liu [16] introduced the definition of fuzzy ring and discussed fuzzy subrings and fuzzy ideals and presented some basic concepts of fuzzy algebra, as fuzzy invariant subgroups, fuzzy ideals 
and proved some related properties. The notion of intuitionistic fuzzy set was introduced by Atanassov [5,6] as a generalization of fuzzy sets. After that many researches applied this notion in various branches of mathematics especially in algebra and defined the notions of intuitionistic fuzzy subgroups, intuitionistic fuzzy subrings and intuitionistic fuzzy normal subgroups. In 1989 [12], Biswas defined the notion of intuitionistic fuzzy groups and investigated its properties. Followed by a work in 2003 by Hur et al. in [14], which introduced the concepts of intuitionistic fuzzy subgroups and intuitionistic fuzzy subrings and examined some of their characteristics. In the same year, Banerjee and Basnet in [9], studied intuitionistic fuzzy subrings and ideals based on intuitionistic fuzzy sets. The notion of intuitionistic fuzzy normed rings was introduced by Abed Alhaleem and Ahmad in [1], in the paper they introduced the concepts of intuitionistic fuzzy normed subrings and intuitionistic fuzzy normed ideals as an extension of fuzzy normed rings which were studied by Emniyet and Sahin [13], which presented the notions of fuzzy normed subrings and fuzzy normed ideals. In 2011, Azam et al. [7] defined anti fuzzy ideal and lower level ideals of a ring and some related properties were proved. Sharma and Bansal [19] followed the work of Azam and introduced the notion of intuitionistic anti fuzzy subrings and intuitionistic anti fuzzy ideals and investigated various characteristics. In this paper, we introduce the notion of intuitionistic anti fuzzy normed subrings and intuitionistic anti fuzzy normed ideals. Further we characterize various properties related to intuitionistic fuzzy normed ideals and identify the anti image of intuitionistic anti fuzzy normed ideals.

\section{Preliminaries}

In this section, we state some basic definitions and results necessary for this paper.

Definition 2.1. [15] A normed ring NR is a ring that possesses a norm \|\| , that is a non-negative real-valued function \|\|$: N R \rightarrow \mathbb{R}$ such that for all $v, r \in N R$, satisfies the following conditions:

(i) $\|v\|=0 \Leftrightarrow v=0 \quad$ (ii) $\|v+r\| \leq\|v\|+\|r\| \quad$ (iii) $\|v r\| \leq\|v\|\|r\|$ and,

(iv) $\|v\|=\|-v\|$ (and thus $\left\|1_{A}\right\|=1=\|-1\|$ if identity exists).

Definition 2.2. [5] Let a set $X$ be fixed. An intuitionistic fuzzy set $A$ is an object having the form

$$
A=\left\{\left(v, \vartheta_{A}(v), \zeta_{A}(v)\right): v \in X\right\}
$$

where the functions $\vartheta_{A}(v): X \rightarrow[0,1]$ and $\zeta_{A}(v): X \rightarrow[0,1]$ denote the degree of membership and the degree of nonmembership, respectively, where $0 \leq \vartheta_{A}(v)+\zeta_{A}(v) \leq 1$ for all $v \in X$.

An intuitionistic fuzzy set $A$ is written symbolically in the form $A=\left(\vartheta_{A}, \zeta_{A}\right)$ [8].

Definition 2.3. [2] Let $*$ be a continuous $t$-norm and $\diamond$ be a continuous s-norm. An intuitionistic fuzzy set $A=\left\{\left(v, \vartheta_{A}(v), \zeta_{A}(v)\right): v \in N R\right\}$ is called an intuitionistic fuzzy normed subring (IFNSR) of the normed ring $(N R,+,$.$) if it satisfies the following conditions for all v, r \in N R$ :

i. $\vartheta_{A}(v-r) \geq \vartheta_{A}(v) * \vartheta_{A}(r)$

ii. $\vartheta_{A}(v r) \geq \vartheta_{A}(v) * \vartheta_{A}(r)$, 
iii. $\zeta_{A}(v-r) \leq \zeta_{A}(v) \diamond \zeta_{A}(r)$,

iv. $\zeta_{A}(v r) \leq \zeta_{A}(v) \diamond \zeta_{A}(r)$

Definition 2.4. [1] If the fuzzy set $A=\left(\vartheta_{A}, \zeta_{A}\right)$ is both right and left intuitionistic fuzzy normed ideal of $N R$, then $A$ is called intuitionistic fuzzy normed ideal (IFNI), if for every $v, r \in N R$ :

i. $\vartheta_{A}(v-r) \geq \vartheta_{A}(v) * \vartheta_{A}(r)$

ii. $\vartheta_{A}(v r) \geq \vartheta_{A}(v) \diamond \vartheta_{A}(r)$,

iii. $\zeta_{A}(v-r) \leq \zeta_{A}(v) \diamond \zeta_{A}(r)$,

iv. $\zeta_{A}(v r) \leq \zeta_{A}(v) * \zeta_{A}(r)$

Proposition 2.5. [1] Let $A$ be an intuitionistic fuzzy normed ring and let 0 be the zero of the normed ring $N R$, then the following is true for every $v \in N R$ :

i. $\vartheta_{A}(v) \leq \vartheta_{A}(0), \zeta_{A}(v) \geq \zeta_{A}(0)$

ii. $\vartheta_{A}(v)=\vartheta_{A}(-v), \zeta_{A}(v)=\zeta_{A}(-v)$.

Lemma 2.6. [1] Let $1_{N R}$ be the multiplicative identity of $N R$ then for all $v \in N R$ :

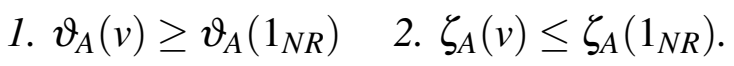

Definition 2.7. [18] Let $A$ be an intuitionistic fuzzy set in a ring $N R$. The set $A_{\alpha, \beta}=\{v: v \in N R \mid$ $\vartheta_{A} \geq \alpha$ and $\left.\zeta_{A} \leq \beta\right\}$ is called the level subset of $A$ where $\alpha+\beta \leq 1$ and $\alpha, \beta \in[0,1]$.

Definition 2.8. [4] If A and B are two intuitionistic anti fuzzy normed subrings. Then we identify:

i. $A^{c}=\left\{\left(v, \zeta_{A}(v), \vartheta_{A}(v)\right): v \in N R\right\}$ and denote by $A^{c}=\left(\zeta_{A}, \vartheta_{A}\right)$.

ii. $A \cap B=\left(\vartheta_{A \cap B}, \zeta_{A \cap B}\right)$ where $\vartheta_{A \cap B}(v)=\left\{\left(v, \max \left(\vartheta_{A}(v), \vartheta_{B}(v)\right)\right): v \in N R\right\}$ and $\zeta_{A \cap B}(v)=$ $\left\{\left(v, \min \left(\zeta_{A}(v), \zeta_{B}(v)\right)\right): v \in N R\right\}$.

iii. $A \cup B=\left(\vartheta_{A \cup B}, \zeta_{A \cup B}\right)$ where $\vartheta_{A \cup B}(v)=\left\{\left(v, \min \left(\vartheta_{A}(v), \vartheta_{B}(v)\right)\right): v \in N R\right\}$ and $\zeta_{A \cup B}(v)=$ $\left\{\left(v, \max \left(\zeta_{A}(v), \zeta_{B}(v)\right)\right): v \in N R\right\}$.

Theorem 2.9. [10] Let $f: R \rightarrow R^{\prime}$ be a surjective ring homomorphism, then

1. If $B$ is IFI in $R^{\prime}$, then $f^{-1}(B)$ is IFI in $R$.

2. If $A$ is IFI in $R$, then $f(A)$ is IFI in $R^{\prime}$.

\section{Intuitionistic anti fuzzy normed ideals}

In this section, we introduce the notions of intuitionistic anti fuzzy normed subrings and intuitionistic anti fuzzy normed ideals. Further, we generalize some basic related properties. 
Definition 3.1. An IFS A of a normed ring NR is an intuitionistic anti fuzzy normed subring (IAFNSR) of NR if it satisfies the following for all $v, r \in N R$ :
i. $\vartheta_{A}(v-r) \leq \vartheta_{A}(v) \diamond \vartheta_{A}(r)$,
ii. $\vartheta_{A}(v r) \leq \vartheta_{A}(v) \diamond \vartheta_{A}(r)$,
iii. $\zeta_{A}(v-r) \geq \zeta_{A}(v) * \zeta_{A}(r)$,
iv. $\zeta_{A}(v r) \geq \zeta_{A}(v) * \zeta_{A}(r)$.

Theorem 3.2. Let $A=\left(\vartheta_{A}, \zeta_{A}\right)$ be an intuitionistic anti fuzzy normed subring of $N R$, then for every $v, r \in N R$ :
i. If $\vartheta_{A}(v) \neq \vartheta_{A}(r)$, then $\vartheta_{A}(v-r)=\vartheta_{A}(v) \diamond \vartheta_{A}(r)$,
ii. If $\zeta_{A}(v) \neq \zeta_{A}(r)$, then $\zeta_{A}(v-r)=\zeta_{A}(v) * \zeta_{A}(r)$.

Proof. $i$. As $\vartheta_{A}(v) \neq \vartheta_{A}(r)$, we assume that $\vartheta_{A}(v)<\vartheta_{A}(r)=\delta$. As $\vartheta_{A}(v-r) \leq \vartheta_{A}(v) \diamond \vartheta_{A}(r) \leq$ $\delta$, then $\vartheta_{A}(v-r) \leq \delta$.

Suppose that $\vartheta_{A}(v-r)<\delta$, so $\vartheta_{A}(r)=\vartheta_{A}(v-(v-r)) \leq \vartheta_{A}(v) \diamond \vartheta_{A}(v-r)<\delta$, hence, $\vartheta_{A}(r)<\delta$, which is a contradiction, so $\vartheta_{A}(v-r)=\delta=\vartheta_{A}(v) \diamond \vartheta_{A}(r)$.

ii. Similar to the proof of $i$.

Remark 3.3. If $A$ is an intuitionistic anti fuzzy normed subring of $N R$, then:

1. Among $\vartheta_{A}(v-r), \vartheta_{A}(v)$ and $\vartheta_{A}(r)$ at least two are equal, and

2. Among $\zeta_{A}(v-r), \zeta_{A}(v)$ and $\zeta_{A}(r)$ at least two are equal.

Proposition 3.4. An IFS A is an intuitionistic anti fuzzy normed subring if and only if $A^{c}$ is an intuitionistic fuzzy normed subring of $N R$.

Proof. Suppose that $A$ is an IAFNSR, we need to show that $A^{c}=\left(\vartheta_{A^{c}}, \zeta_{A^{c}}\right)=\left(\zeta_{A}, \vartheta_{A}\right)$ is an intuitionistic fuzzy normed subring of $N R$.

$i$.

$$
\begin{aligned}
\vartheta_{A}^{c}(v-r) & =1-\vartheta_{A}(v-r) \\
& \geq 1-\left(\vartheta_{A}(v) \diamond \vartheta_{A}(r)\right) \\
& \geq 1-\max \left(\vartheta_{A}(v), \vartheta_{A}(r)\right) \\
& =\min \left(1-\vartheta_{A}(v), 1-\vartheta_{A}(r)\right) \\
& =\min \left(\vartheta_{A}^{c}(v), \vartheta_{A}^{c}(r)\right) .
\end{aligned}
$$

Then, $\vartheta_{A}^{c}(v-r) \geq \vartheta_{A}^{c}(v) * \vartheta_{A}^{c}(r)$.

ii.

$$
\begin{aligned}
\vartheta_{A}^{c}(v r) & =1-\vartheta_{A}(v r) \\
& \geq 1-\left(\vartheta_{A}(v) \diamond \vartheta_{A}(r)\right) \\
& \geq 1-\max \left(\vartheta_{A}(v), \vartheta_{A}(r)\right) \\
& =\min \left(1-\vartheta_{A}(v), 1-\vartheta_{A}(r)\right) \\
& =\min \left(\vartheta_{A}^{c}(v), \vartheta_{A}^{c}(r)\right) .
\end{aligned}
$$

Then, $\vartheta_{A}^{c}(v r) \geq \vartheta_{A}^{c}(v) * \vartheta_{A}^{c}(r)$. 
iii.

$$
\begin{aligned}
\zeta_{A}^{c}(v-r) & =1-\zeta_{A}(v-r) \\
& \leq 1-\left(\zeta_{A}(v) \diamond \zeta_{A}(r)\right) \\
& \leq 1-\min \left(\zeta_{A}(v), \zeta_{A}(r)\right) \\
& =\max \left(1-\zeta_{A}(v), 1-\zeta_{A}(r)\right) \\
& =\max \left(\zeta_{A}^{c}(v), \zeta_{A}^{c}(r)\right)
\end{aligned}
$$

Then, $\zeta_{A}^{c}(v-r) \leq \zeta_{A}^{c}(v) \diamond \zeta_{A}^{c}(r)$.

$i v$.

$$
\begin{aligned}
\zeta_{A}^{c}(v r) & =1-\zeta_{A}(v r) \\
& \leq 1-\left(\zeta_{A}(v) \diamond \zeta_{A}(r)\right) \\
& \leq 1-\min \left(\zeta_{A}(v), \zeta_{A}(r)\right) \\
& =\max \left(1-\vartheta_{A}(v), 1-\zeta_{A}(r)\right) \\
& =\max \left(\zeta_{A}^{c}(v), \zeta_{A}^{c}(r)\right) .
\end{aligned}
$$

Then, $\zeta_{A}^{c}(v r) \leq \zeta_{A}^{c}(v) \diamond \zeta_{A}^{c}(r)$.

On the other hand, we can prove similarly that if $A^{c}$ is an intuitionistic fuzzy normed subring, then $A$ is an IAFNSR of $N R$.

Definition 3.5. An intuitionistic fuzzy set A of a normed ring NR is said to be an intuitionistic anti fuzzy normed left (right) ideal of $N R$ if for all $v, r \in N R$ :
i. $\vartheta_{A}(v-r) \leq \vartheta_{A}(v) \diamond \vartheta_{A}(r)$
ii. $\vartheta_{A}(v r) \leq \vartheta_{A}(r)\left(\vartheta_{A}(v r) \leq \vartheta_{A}(v)\right)$,
iii. $\zeta_{A}(v-r) \geq \zeta_{A}(v) * \zeta_{A}(r)$,
iv. $\zeta_{A}(v r) \geq \zeta_{A}(r)\left(\zeta_{A}(v r) \geq \zeta_{A}(v)\right)$.

Definition 3.6. An IFS A of a normed ring NR is said to be an intuitionistic anti fuzzy normed ideal (IAFNI) of NR if it satisfies the following conditions for all $v, r \in N R$ :
i. $\vartheta_{A}(v-r) \leq \vartheta_{A}(v) \diamond \vartheta_{A}(r)$
ii. $\vartheta_{A}(v r) \leq \vartheta_{A}(v) * \vartheta_{A}(r)$,
iii. $\zeta_{A}(v-r) \geq \zeta_{A}(v) * \zeta_{A}(r)$,
iv. $\zeta_{A}(v r) \geq \zeta_{A}(v) \diamond \zeta_{A}(r)$.

Proposition 3.7. An IFS A is an intuitionistic anti fuzzy normed ideal if and only if $A^{c}$ is an intuitionistic fuzzy normed ideal of $N R$.

Proof. Suppose that $A$ is an IAFNI, we need to show that $A^{c}=\left(\vartheta_{A^{c}}, \zeta_{A^{c}}\right)=\left(\zeta_{A}, \vartheta_{A}\right)$ is an intuitionistic fuzzy normed ideal of $N R$. 
$i$.

$$
\begin{aligned}
& \vartheta_{A}(v-r) \leq \vartheta_{A}(v) \diamond \vartheta_{A}(r) \Rightarrow \zeta_{A}^{c}(v-r) \leq \zeta_{A}^{c}(v) \diamond \zeta_{A}^{c}(r) \\
& \Rightarrow 1-\vartheta_{A}^{c}(v-r) \leq\left\{1-\vartheta_{A}^{c}(v)\right\} \diamond\left\{1-\vartheta_{A}^{c}(r)\right\} \Rightarrow 1-\vartheta_{A}^{c}(v-r) \leq 1-\left\{\vartheta_{A}^{c}(v) * \vartheta_{A}^{c}(r)\right\} .
\end{aligned}
$$

Therefore, $\vartheta_{A}^{c}(v-r) \geq \vartheta_{A}^{c}(v) * \vartheta_{A}^{c}(r)$.

ii. Similarly we can show $\vartheta_{A}^{c}(v r) \geq \vartheta_{A}^{c}(v) \diamond \vartheta_{A}^{c}(r)$.

iii.

$$
\begin{aligned}
& \zeta_{A}(v-r) \geq \zeta_{A}(v) * \zeta_{A}(r) \Rightarrow \vartheta_{A}^{c}(v-r) \geq \vartheta_{A}^{c}(v) * \vartheta_{A}^{c}(r) \\
& \Rightarrow 1-\zeta_{A}^{c}(v-r) \geq\left\{1-\zeta_{A}^{c}(v)\right\} *\left\{1-\zeta_{A}^{c}(r)\right\} \Rightarrow 1-\zeta_{A}^{c}(v-r) \geq 1-\left\{\zeta_{A}^{c}(v) \diamond \zeta_{A}^{c}(r)\right\} .
\end{aligned}
$$

Therefore, $\zeta_{A}^{c}(v-r) \leq \zeta_{A}^{c}(v) \diamond \zeta_{A}^{c}(r)$.

$i v$. Similarly we can show $\zeta_{A}^{c}(v r) \leq \zeta_{A}^{c}(v) * \zeta_{A}^{c}(r)$.

On the other hand, we can prove similarly that if $A^{c}$ is an intuitionistic fuzzy normed ideal, then $A$ is an $I A F N I$ of $N R$.

Theorem 3.8. Let $A$ and $B$ be two intuitionistic anti fuzzy ideals of a normed ring $N R$. Then $A \cap B$ is an intuitionistic anti fuzzy normed ideal of $N R$.

Proof. Let $v, r \in N R$. Then,

$i$.

$$
\begin{aligned}
\vartheta_{A \cap B}(v-r) & =\max \left(\vartheta_{A}(v-r), \vartheta_{B}(v-r)\right) \\
& \leq \max \left(\vartheta_{A}(v) \diamond \vartheta_{A}(r), \vartheta_{B}(v) \diamond \vartheta_{B}(r)\right) \\
& \leq \max \left(\left(\vartheta_{A}(v) \diamond \vartheta_{B}(v)\right),\left(\vartheta_{A}(r) \diamond \vartheta_{B}(r)\right)\right) \\
& \leq \max \left(\vartheta_{A \cap B}(v), \vartheta_{A \cap B}(r)\right) \\
& \leq \vartheta_{A \cap B}(v) \diamond \vartheta_{A \cap B}(r)
\end{aligned}
$$

ii.

$$
\begin{aligned}
\vartheta_{A \cap B}(v r) & =\max \left(\vartheta_{A}(v r), \vartheta_{B}(v r)\right) \\
& \leq \max \left(\vartheta_{A}(r), \vartheta_{B}(r)\right) \\
& \leq \vartheta_{A \cap B}(r)
\end{aligned}
$$

iii.

$$
\begin{aligned}
\zeta_{A \cap B}(v-r) & =\min \left(\zeta_{A}(v-r), \zeta_{B}(v-r)\right) \\
& \geq \min \left(\zeta_{A}(v) * \zeta_{A}(r), \zeta_{B}(v) * \zeta_{B}(r)\right) \\
& \geq \min \left(\left(\zeta_{A}(v) * \zeta_{B}(v)\right),\left(\zeta_{A}(r) * \zeta_{B}(r)\right)\right) \\
& \geq \min \left(\zeta_{A \cap B}(v), \zeta_{A \cap B}(r)\right\} \\
& \geq \zeta_{A \cap B}(v) * \zeta_{A \cap B}(r)
\end{aligned}
$$

$i v$.

$$
\begin{aligned}
\zeta_{A \cap B}(v r) & =\min \left(\zeta_{A}(v r), \zeta_{B}(v r)\right) \\
& \geq \min \left(\zeta_{A}(r), \zeta_{B}(r)\right) \\
& \geq \zeta_{A \cap B}(r)
\end{aligned}
$$

Hence, $A \cap B$ is an intuitionistic anti fuzzy normed left ideal. Likewise it can be proven that $A \cap B$ is an intuitionistic anti fuzzy normed right ideal. So, $A \cap B$ is an IAFNI of $N R$.

Remark 3.9. The union of two intuitionistic anti fuzzy normed ideals of a ring NR needs not be always intuitionistic anti fuzzy normed ideal. 
Example 3.1. Let $N R=\mathbb{Z}$ be the ring of integers under ordinary addition and multiplication of integers.Let the intuitionistic anti fuzzy normed subsets $A=\left(\vartheta_{A}, \zeta_{A}\right)$ and $B=\left(\vartheta_{B}, \zeta_{B}\right)$, be defined by

$$
\begin{aligned}
& \vartheta_{A}(v)=\left\{\begin{array}{cl}
0.35 & , \text { if } v \in 3 \mathbb{Z} \\
1 & \text {, otherwise }
\end{array} \text { and } \zeta_{A}(v)=\left\{\begin{array}{cl}
0.25 & , \text { if } v \in 3 \mathbb{Z} \\
0 & \text {, otherwise }
\end{array}\right.\right. \\
& \vartheta_{B}(v)=\left\{\begin{array}{cl}
0.7 & , \text { if } v \in 2 \mathbb{Z} \\
0.85 & \text {, otherwise }
\end{array} \text { and } \quad \zeta_{B}(v)=\left\{\begin{array}{cl}
0.1 & \text {, if } v \in 2 \mathbb{Z} \\
0.03 & \text {, otherwise }
\end{array}\right.\right.
\end{aligned}
$$

It can be checked that $A$ and $B$ are IAFNI of $\mathbb{Z}$.

As $\vartheta_{A \cup B}(v)=\min \left(\vartheta_{A}(v), \vartheta_{B}(v)\right)$ and $\zeta_{A \cup B}(v)=\max \left(\zeta_{A}(v), \zeta_{B}(v)\right)$. Then,

$$
\vartheta_{A \cup B}(v)=\left\{\begin{array}{cl}
0.35 & , \text { if } v \in 3 \mathbb{Z} \\
0.7 & , \text { if } v \in 2 \mathbb{Z}-3 \mathbb{Z} \\
0.85 & , \text { if } v \notin 2 \mathbb{Z} \text { or } v \notin 3 \mathbb{Z}
\end{array} \quad \text { and } \quad \zeta_{A \cup B}(v)=\left\{\begin{array}{cl}
0.25 & , \text { if } v \in 3 \mathbb{Z} \\
0.1 & \text {, if } v \in 2 \mathbb{Z}-3 \mathbb{Z} \\
0.03 & \text {, if } v \notin 2 \mathbb{Z} \text { or } v \notin 3 \mathbb{Z}
\end{array}\right.\right.
$$

Let $v=9$ and $r=2$, then $\vartheta_{A \cup B}(9)=0.35, \vartheta_{A \cup B}(2)=0.7$ and $\zeta_{A \cup B}(9)=0.25, \zeta_{A \cup B}(2)=0.1$. Hence, $\vartheta_{A \cup B}(9-2)=\vartheta_{A \cup B}(7)=0.85 \nless \vartheta_{A \cup B}(9) \diamond \vartheta_{A \cup B}(2)=\max (0.35,0.7)$ and $\zeta_{A \cup B}(9-2)=$ $\zeta_{A \cup B}(7)=0.03 \ngtr \zeta_{A \cup B}(9) * \zeta_{A \cup B}(2)=\min (0.25,0.1)$. Thus, the union of two IAFNI's of NR need not be an IAFNI.

Proposition 3.10. Let $A$ be an intuitionistic anti fuzzy normed ideal of a ring $N R$, then we have for all $v \in N R$ :

i. $\vartheta_{A}(0) \leq \vartheta_{A}(v)$ and $\zeta_{A}(0) \geq \zeta_{A}(v)$.

ii. $\vartheta_{A}(-v)=\vartheta_{A}(v)$ and $\zeta_{A}(-v)=\zeta_{A}(v)$.

iii. if $\vartheta_{A}(v-r)=\vartheta_{A}(0)$, then $\vartheta_{A}(v)=\vartheta_{A}(r)$.

iv. if $\zeta_{A}(v-r)=\zeta_{A}(0)$, then $\zeta_{A}(v)=\zeta_{A}(r)$.

v. $\vartheta_{A}(v) \leq \vartheta_{A}\left(1_{N R}\right)$ and $\zeta_{A}(v) \geq \zeta_{A}\left(1_{N R}\right)$, if identity exists.

Proof. $i$. As $A$ is an IAFNI, then

$$
\vartheta_{A}(0)=\vartheta_{A}(v-v) \leq \vartheta_{A}(v) \diamond \vartheta_{A}(v)=\vartheta_{A}(v)
$$

and

$$
\zeta_{A}(0)=\zeta_{A}(v-v) \geq \zeta_{A}(v) * \zeta_{A}(v)=\zeta_{A}(v) .
$$

ii. $\vartheta_{A}(-v)=\vartheta_{A}(0-v) \leq \vartheta_{A}(0) \diamond \vartheta_{A}(v)=\vartheta_{A}(v)$ and $\vartheta_{A}(v)=\vartheta_{A}(0-(-v)) \leq \vartheta_{A}(0) \diamond \vartheta_{A}(-v)=$ $\vartheta_{A}(-v)$.

Also, $\zeta_{A}(-v)=\zeta_{A}(0-v) \geq \zeta_{A}(0) * \zeta_{A}(v)=\zeta_{A}(v)$ and $\zeta_{A}(v)=\zeta_{A}(0-(-v)) \geq \zeta_{A}(0) * \zeta_{A}(-v)=$ $\zeta_{A}(-v)$. 
iii. Since $\vartheta_{A}(v-r)=\vartheta_{A}(0)$, then

$$
\vartheta_{A}(r)=\vartheta_{A}(v-(v-r)) \leq \vartheta_{A}(v) \diamond \vartheta_{A}(v-r)=\vartheta_{A}(v) \diamond \vartheta_{A}(0) \leq \vartheta_{A}(v)
$$

Similarly,

$$
\vartheta_{A}(v)=\vartheta_{A}((v-r)-(-r)) \leq \vartheta_{A}(v-r) \diamond \vartheta_{A}(-r)=\vartheta_{A}(0) \diamond \vartheta_{A}(r) \leq \vartheta_{A}(r)
$$

Consequently, $\vartheta_{A}(v)=\vartheta_{A}(r)$.

iv. Same as in iii.

v. $\vartheta_{A}(v)=\vartheta_{A}\left(v \cdot 1_{N R}\right) \leq \vartheta_{A}(v) * \vartheta_{A}\left(1_{N R}\right)$, then $\vartheta_{A}(v) \leq \vartheta_{A}\left(1_{N R}\right)$. Similarly, $\zeta_{A}(v)=\zeta_{A}\left(v \cdot 1_{N R}\right) \geq$ $\zeta_{A}(v) \diamond \zeta_{A}\left(1_{N R}\right)$, then $\zeta_{A}(v) \geq \zeta_{A}\left(1_{N R}\right)$.

Proposition 3.11. If $A$ is an IAFNI of $N R$, then $A_{*}=\left\{v \in N R: \vartheta_{A}(v)=\vartheta_{A}\left(0_{N R}\right)\right.$ and $\zeta_{A}(v)=$ $\left.\zeta_{A}\left(0_{N R}\right)\right\}$ is a left (right) ideal of $N R$.

Proof. Let $v, r \in A_{*}$, then $\vartheta_{A}(v)=\vartheta_{A}(r)=\vartheta_{A}(0)$ and $\zeta_{A}(v)=\zeta_{A}(r)=\zeta_{A}(0)$. Since $A$ is an $I A F N I$ of $N R$, then

$\vartheta_{A}(v-r) \leq \vartheta_{A}(v) \diamond \vartheta_{A}(r)=\vartheta_{A}(0) \diamond \vartheta_{A}(0)=\vartheta_{A}(0)$

and

$\zeta_{A}(v-r) \geq \zeta_{A}(v) * \zeta_{A}(r)=\zeta_{A}(0) * \zeta_{A}(0)=\zeta_{A}(0)$

Hence, $\vartheta_{A}(v-r)=\vartheta_{A}(0)$ and $\zeta_{A}(v-r)=\zeta_{A}(0)$. Thus, $v-r \in A_{*}$.

Let $z \in N R$ and $v \in A_{*}$. We have,

$\vartheta_{A}(z v) \leq \vartheta_{A}(z) * \vartheta_{A}(v)=\vartheta_{A}(0)$ and $\zeta_{A}(z v) \geq \zeta_{A}(z) \diamond \zeta_{A}(v)=\zeta_{A}(0)$.

This implies that, $\vartheta_{A}(z v)=\vartheta_{A}(0)$ and $\zeta_{A}(z v)=\zeta_{A}(0)$. Therefore, $z v \in A_{*}$. Thus, $A_{*}$ is a left ideal of $N R$.

Definition 3.12. Let $A$ be an intuitionistic fuzzy set in a ring $N R$. The set $\bar{A}_{\alpha, \beta}=\left\{v \in N R: \vartheta_{A} \leq \alpha\right.$ and $\left.\zeta_{A} \geq \beta\right\}$ is called anti level subset of $A$ where $\alpha+\beta \leq 1$ and $\alpha, \beta \in[0,1]$.

Theorem 3.13. Let $A$ be an intuitionistic fuzzy set in a ring NR. If $A$ is an intuitionistic anti fuzzy left (right) ideal of $N R$, then each anti level subset $\bar{A}_{\alpha, \beta}$, is a left (right) ideal of $N R$.

Proof. Let $A$ be an IAFNI in a ring NR. Let $v, r \in \bar{A}_{\alpha, \beta}$, then we have $\vartheta_{A}(v) \leq \alpha, \vartheta_{B}(r) \leq \alpha$ and $\zeta_{A}(v) \geq \beta, \zeta_{A}(r) \geq \beta$

Since $A$ is an IAFNI of $N R$, then $\vartheta_{A}(v-r) \leq \vartheta_{A}(v) \diamond \vartheta_{A}(r) \leq \alpha$ and $\zeta_{A}(v-r) \geq \zeta_{A}(v) * \zeta_{A}(r) \geq \beta$. Hence, $\vartheta_{A}(v-r) \leq \alpha$ and $\zeta_{A}(v-r) \geq \beta$. Thus, $v-r \in \bar{A}_{\alpha, \beta}$.

Let $z \in N R$ and $v \in \bar{A}_{\alpha, \beta}$. Then we have, $\vartheta_{A}(z v) \leq \vartheta_{A}(v) \leq \alpha$ and $\zeta_{A}(z v) \geq \zeta_{A}(v) \geq \beta$.

This implies that, $\vartheta_{A}(z v) \leq \alpha$ and $\zeta_{A}(z v) \geq \beta$. Therefore, $z v \in \bar{A}_{\alpha, \beta}$. Thus, $\bar{A}_{\alpha, \beta}$ is a left ideal of $N R$.

Definition 3.14. [1] Let $N R$ and $N R^{\prime}$ be normed rings and let $f: N R \rightarrow N R^{\prime}$ be a ring homomorphism. If $A=\left(\vartheta_{A}, \zeta_{A}\right)$ and $B=\left(\vartheta_{B}, \zeta_{B}\right)$ are intuitionistic fuzzy normed subrings of $N R$ and $N R^{\prime}$, respectively, then $f(A)=\left\{\left(r, \vartheta_{f(A)}(r), \zeta_{f(A)}(r)\right): r \in N R^{\prime}\right\}$ is the intuitionistic image of $A$, where 
$\vartheta_{f(A)}(r)$ and $\zeta_{f(A)}(r)$ are specified for all $r \in N R^{\prime}$ as:

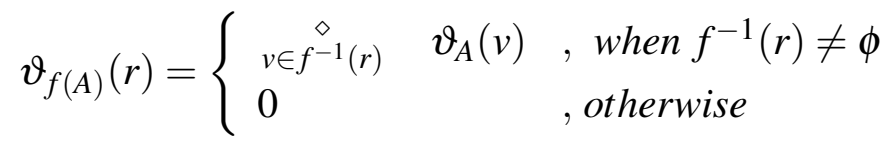

and

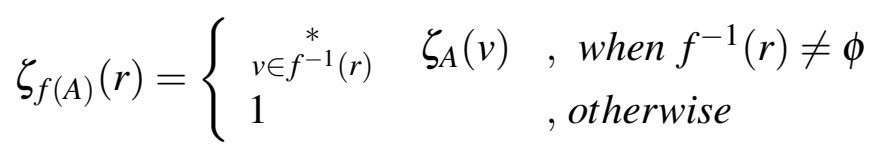

Definition 3.15. Let $f: N R \rightarrow N R^{\prime}$ be a ring homomorphism. If $A$ is an intuitionistic fuzzy normed subring in $N R$, then the anti image of $A$ under $f$ is the intuitionistic fuzzy normed subring $f_{-}(A)=\left\{\left(r, \vartheta_{f_{-}(A)}(r), \zeta_{f_{-}(A)}(r)\right): r \in N R^{\prime}\right\}$, where $\vartheta_{f_{-}(A)}(r)$ and $\zeta_{f_{-}(A)}(r)$ are specified for all $r \in N R^{\prime}$ as:

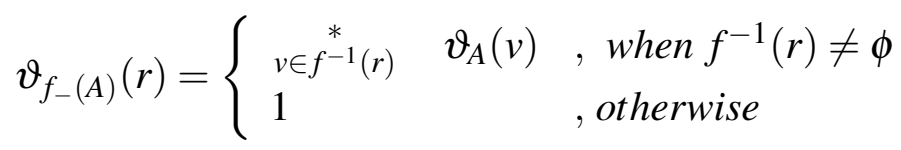

and

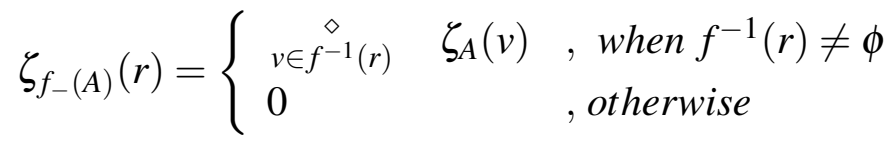

Definition 3.16. [1] Let $f: N R \rightarrow N R^{\prime}$ be a ring homomorphism and $B=\left(\vartheta_{B}, \zeta_{B}\right)$ be an intuitionistic fuzzy normed subring of $N R^{\prime}$. Then the inverse image

$$
f^{-1}(B)=\left\{\left(v, \vartheta_{f^{-1}(B)}(v), \zeta_{f^{-1}(B)}(v)\right): v \in N R\right\}
$$

is an intuitionistic fuzzy normed subring in NR such that $f^{-1}(B)(v)=B(f(v))$, where $\vartheta_{f^{-1}(B)}(v)=$ $\vartheta_{B}(f(v))$ and $\zeta_{f^{-1}(B)}(v)=\zeta_{B}(f(v))$.

Theorem 3.17. A homomorphic inverse image of an intuitionistic anti fuzzy normed ideal is an intuitionistic anti fuzzy normed ideal.

Proof. Let $f: N R \rightarrow N R^{\prime}$ be a ring homomorphism. Let $B$ be an IAFNI in $N R^{\prime}$ and $f^{-1}$ be the inverse image of $B$. Suppose $v, r \in N R$, then we have:

$i$.

$$
\begin{aligned}
\vartheta_{f^{-1}(B)}(v-r) & =\vartheta_{B}(f(v-r)) \\
& =\vartheta_{B}(f(v)-f(r)) \\
& \leq \vartheta_{B}(f(v)) \diamond \vartheta_{B}(f(r)) \\
& \leq \vartheta_{f^{-1}(B)}(v) \diamond \vartheta_{f^{-1}(B)}(r)
\end{aligned}
$$

ii.

$$
\begin{aligned}
\vartheta_{f^{-1}(B)}(v r) & =\vartheta_{B}(f(v r)) \\
& =\vartheta_{B}(f(v) f(r)) \\
& \leq \vartheta_{B}(f(v)) * \vartheta_{B}(f(r)) \\
& \leq \vartheta_{f^{-1}(B)}(v) * \vartheta_{f^{-1}(B)}(r) .
\end{aligned}
$$


iii.

$$
\begin{aligned}
\zeta_{f^{-1}(B)}(v-r) & =\zeta_{B}(f(v-r)) \\
& =\zeta_{B}(f(v)-f(r)) \\
& \geq \zeta_{B}(f(v)) * \zeta_{B}(f(r)) \\
& \geq \zeta_{f^{-1}(B)}(v) * \zeta_{f^{-1}(B)}(r) .
\end{aligned}
$$

$i v$.

$$
\begin{aligned}
\zeta_{f^{-1}(B)}(v r) & =\zeta_{B}(f(v r)) \\
& =\zeta_{B}(f(v) f(r)) \\
& \geq \zeta_{B}(f(v)) \diamond \zeta_{B}(f(r)) \\
& \geq \zeta_{f^{-1}(B)}(v) \diamond \zeta_{f^{-1}(B)}(r) .
\end{aligned}
$$

Hence, $f^{-1}(B)$ is an IAFNI of $N R$.

Theorem 3.18. Let $f: N R \rightarrow N R^{\prime}$ be a surjective ring homomorphism. Then:

i. If $B$ is an intuitionistic fuzzy set in $N R^{\prime}$, then $f^{-1}\left(B^{c}\right)=\left(f^{-1}(B)\right)^{c}$.

ii. If $A$ is an intuitionistic fuzzy set in $N R$, then $f\left(A^{c}\right)=\left(f_{-}(A)\right)^{c}$.

Proof. $i$. As $B$ is an intuitionistic fuzzy set in $N R^{\prime}$, then we have for every $v \in N R$ :

$f^{-1}\left(B^{c}\right)(v)=B^{c}(f(v))=\left(\zeta_{B}(f(v)), \vartheta_{B}(f(v))\right)=\left(\zeta_{f^{-1}(B)}(v), \vartheta_{f^{-1}(B)}(v)\right)=\left(f^{-1}(B)\right)^{c}(v)$.

ii. As $A$ is an intuitionistic fuzzy set in $N R$, then we have for every $r \in N R^{\prime}$ :

$$
\begin{aligned}
& f\left(A^{c}\right)(r)=\left(\underset{v \in f^{-1}(r)}{\diamond} \quad \vartheta_{A^{c}}(v), \underset{v \in f^{-1}(r)}{*} \quad \zeta_{A^{c}}(v)\right) \\
& =\left(\underset{v \in f^{-1}(r)}{\diamond} \quad \zeta_{A}(v), \underset{v \in f^{-1}(r)}{*} \quad \vartheta_{A}(v)\right) \\
& =\left(f_{-}(A)\right)^{c}(r) \text {. }
\end{aligned}
$$

Theorem 3.19. Let $f: N R \rightarrow N R^{\prime}$ be a surjective ring homomorphism. Then:

i. If $B$ is an intuitionistic anti fuzzy normed ideal of $N R^{\prime}$, then $f^{-1}(B)$ is an intuitionistic anti fuzzy normed ideal of $N R$.

ii. If $A$ is an intuitionistic anti fuzzy normed ideal of $N R$, then $f_{-}(A)$ is an intuitionistic anti fuzzy normed ideal of $N R^{\prime}$.

Proof. $i$. As $B$ is an IAFNI in $N R^{\prime}$, then $B^{c}$ is an intuitionistic fuzzy normed ideal in $N R^{\prime}$ and then $f^{-1}\left(B^{c}\right)$ is an intuitionistic fuzzy normed ideal in $N R$ which means that $\left(f^{-1}(B)\right)^{c}$ is an intuitionistic fuzzy normed ideal in $N R$. Hence, $f^{-1}(B)$ is an IAFNI in $N R$.

ii. As $A$ is an IAFNI in $N R$, then $A^{c}$ is an intuitionistic fuzzy normed ideal in $N R$ and then $f\left(A^{c}\right)$ is an intuitionistic fuzzy normed ideal in $N R^{\prime}$. As $f\left(A^{c}\right)=\left(f_{-}(A)\right)^{c}$ which means that $\left(f_{-}(A)\right)^{c}$ is an intuitionistic fuzzy normed ideal in $N R^{\prime}$. Hence, $f_{-}(A)$ is an IAFNI in $N R^{\prime}$.

\section{References}

[1] Abed Alhaleem, N., \& Ahmad, A. G. (2020). Intuitionistic Fuzzy Normed Subrings and Intuitionistic Fuzzy Normed Ideals. Mathematics, 8, Article ID 1594. 
[2] Abed Alhaleem, N., \& Ahmad, A. G. (2021). Intuitionistic Fuzzy Normal Subrings over Normed Rings. International Journal of Analysis and Applications, 19, 341-359.

[3] Alsarahead, M. O., \& Ahmad, A. G. (2018). Complex intuitionistic fuzzy ideals. AIP Conference Proceedings, Vol. 1940, AIP Publishing LLC, Article ID 020118.

[4] Anitha, B. (2019). Properties of intuitionistic anti fuzzy normal subrings. Malaya Journal of Matematik, 7, 304-308.

[5] Atanassov, K. (1986). Intuitionistic fuzzy sets. Fuzzy Sets and Systems, 20, 87-96.

[6] Atanassov, K. (1999). Intuitionistic Fuzzy Sets: Theory and Applications, Springer, Berlin, Germany.

[7] Azam, F., Mamun, A., \& Nasrin, F. (2013). Anti fuzzy ideal of a ring. Annals of Fuzzy Mathematics and Informatics, 5, 349-360.

[8] Bakhadach, I., Melliani, S., Oukessou, M., \& Chadli, L. S. (2016). Intuitionistic fuzzy ideal and intuitionistic fuzzy prime ideal in a ring. Notes on Intuitionistic Fuzzy Sets, 22(2), 59-63.

[9] Banerjee, B., \& Basnet, D. (2003). Intuitionistic fuzzy subrings and ideals. Journal of Fuzzy Mathematics, 11, 139-155.

[10] Basnet, D. K. (2011). Topics in Intuitionistic Fuzzy Algebra. Lambert Academic Publishing.

[11] Basnet, D. K. (2010). ( $\alpha, \beta)$-cut of intuitionistic fuzzy ideals. International Journal of Algebra, 4, 1329-1334.

[12] Biswas, R. (1989). Intuitionistic fuzzy subgroups. Mathematical Forum, 10, 37-46.

[13] Emniyet, A., \& Şahin, M. (2018). Fuzzy normed rings. Symmetry, 10, 515.

[14] Hur, K., Kang, H. W., \& Song, H. K. (2003). Intuitionistic fuzzy subgroups and subrings. Honam Mathematical Journal, 25, 19-41.

[15] Jarden, M. (2011). Normed rings. Algebraic Patching, Springer, Berlin, Germany, 10-30.

[16] Liu, W. J. (1982). Fuzzy invariant subgroups and fuzzy ideals. Fuzzy Sets Systems, 8, 133-139.

[17] Rosenfeld, A. (1971). Fuzzy invariant subgroups and fuzzy ideals. Journal of Mathematical Analysis and Applications, 35, 512-517.

[18] Santhi, R., \& Kungumaraj, E. (2020). Topologies generated by intuitionistic fuzzy numbers. Notes on Intuitionistic Fuzzy Sets, 26(1), 36-45.

[19] Sharma, P., \& Bansal, V. (2012). On intuitionistic anti fuzzy ideal in rings. International Journal of Mathematical Sciences, 11, 237-243. 
[20] Sharma, P., \& Kaur, G. (2018). On intuitionistic fuzzy prime submodules. Notes on Intuitionistic Fuzzy Sets, 24(4), 97-112.

[21] Zadeh, L. (1965). Fuzzy sets. Information and Control, 8, 338-353.

Editorial remark: The Journal Editors recommend that the authors in their future work use the established notation for the membership function $(\mu)$ and the non-membership function $(v)$ of an intuitionistic fuzzy set. 